\section{NEURAL DEVELOPMENT}

\section{Hands of (cell) fate}

The influence of cellular bioelectric properties, such as the resting membrane potential $\left(\mathrm{V}_{\mathrm{m}}\right)$, on the behaviour of non-excitable cells, such as neural progenitors (APs), is not well understood. Here, Vitali et al. show that as cortical development progresses, the $\mathrm{V}_{\mathrm{m}}$ of APs in the ventricular zone becomes progressively more hyperpolarized and drives the generation of successive subclasses of neurons.

During corticogenesis, subclasses of excitatory neurons are generated sequentially and migrate to populate cortical layers from the inside out, with the peak of layer 4 (L4) neurons being born at embryonic day 14.5 (E14.5). To investigate whether activity manipulation affected neuron differentiation, the authors used in utero electroporation (IUE) on E14.5 in mice to overexpress the inward-rectifying $\mathrm{K}^{+}$channel Kir2.1 in ventricular zone APs, and then looked at how the resulting hyperpolarization affected neuronal identity in primary sensory cortex postnatally. In contrast to controls, E14.5-born L4 neurons in Kir2.1 IUE mice were found in $L 4$ as expected, but also L2/3, which would normally occur only for neurons born at E15.5. These precociously located neurons showed numerous characteristics of L2/3 neurons, including expressing $L 2 / 3$ transcriptional programmes, displaying apical dendrites and symmetrical dendritic arbours, as well as receiving input from L2 neurons and sending callosal projections. Together, these findings suggest that Kir2.1 IUE results in a forward temporal shift in laminar, morphological, molecular and circuit-level characteristics. The authors then investigated whether this identity reassignment was the result of a pre- or post-mitotic effect of hyperpolarization. The temporally selective hyperpolarization of APs replicated the identity shift previously reported. Moreover, whole-cell patch clamp of apical progenitors in E12.5-15.5 cortical slices revealed that there was a sharp drop in the $\mathrm{V}_{\mathrm{m}}$ over this period, similar in magnitude to that induced by Kir2.1 IUE. As corticogenesis progresses, a greater proportion of APs are reported to differentiate into neurons via intermediate progenitors (indirect neurogenesis). Differential labelling of APs, intermediate progenitors and new-born neurons showed that Kir2.1 IUE induced a precocious shift from direct to indirect neurogenesis.

The authors hypothesized that changes in the $V_{m}$ might constitute an endogenous mechanism for regulating neuronal diversity in developing cortex. Using single-cell RNA sequencing, they found that Kir2.1 overexpression in APs at E14.5 repressed genes that are involved in WNT signalling. Temporally selective repression of WNT in APs replicated the effects of Kir2.1 upregulation on neuronal fate. Overall, these findings suggest that membrane hyperpolarization is permissive for specific molecular pathways, including WNT signalling, which in turn coordinate AP developmental programmes and neocortical neuron diversity.

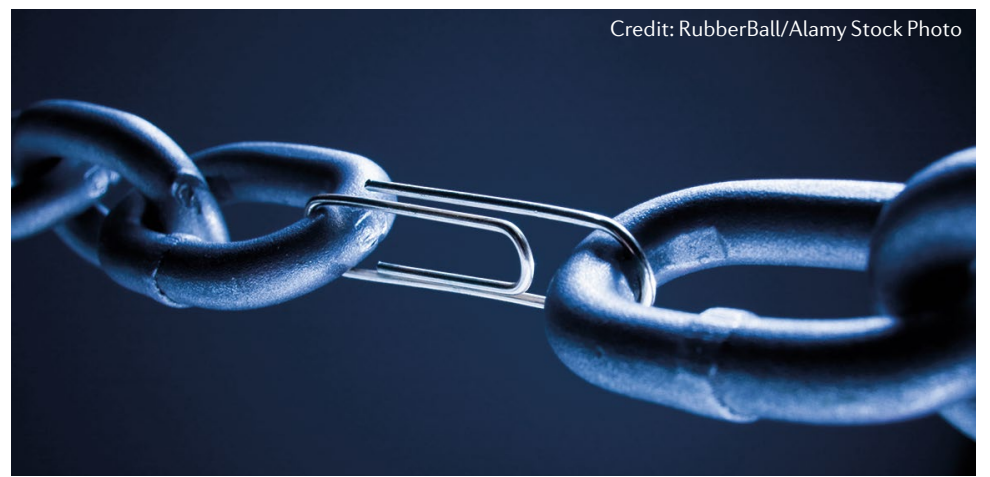

$\Rightarrow$ ADDICTION

\title{
A narcotic-narcoleptic link
}

Hypothalamic neurons that produce hypocretin neuropeptides (also known as orexins) are involved in pleasure-associated arousal and have thus been hypothesized to have a role in opioid addiction. In narcolepsy, a disorder characterized by chronic sleepiness and cataplexy (suddenonset sleep), $\sim 90 \%$ of hypocretin cells are selectively lost. By studying post-mortem human brains and mouse models, Thannickal et al. now identify a striking connection between narcolepsy, opioid addiction and hypocretin cells.

In a previous study of postmortem human brain samples, the authors identified a 'control' brain that exhibited $50 \%$ more hypocretin-expressing cells than in other controls. The authors learned that this individual was formerly addicted to heroin. In the present study, the authors confirmed this upregulation of hypocretin neurons in the brains of five individuals who had been addicted to heroin.

The authors treated mice with morphine to test whether it would induce similar changes. Morphine treatment over 14 days induced a $38 \%$ increase in hypocretin cells that persisted for weeks after cessation of morphine treatment. Immunolabelling for markers of newborn neurons indicated that the increase in hypocretin cell number was not due to neurogenesis, suggesting that opiates may induce hypocretin production in existing neurons. In addition, morphine injected into freely moving, naive rats increased the activity of recorded hypocretin neurons, suggesting that opiates promote hypocretin release.
Next, the authors used a mouse model of narcolepsy in which temporary withdrawal of doxycyline (DOX) causes a selective ablation of hypocretin cells. DOX withdrawal for 1.5 days led to a $30 \%$ loss of hypocretin neurons, but subsequent morphine treatment for 14 days restored the number of hypocretin cells to control levels. Withdrawal of DOX treatment for 18 days led to a loss of $95 \%$ of hypocretin cells and symptoms of narcolepsy, including cataplexy; long-term treatment of these mice with morphine reduced cataplexy.

Last, the authors studied the postmortem brains of two individuals diagnosed with narcolepsy. One of these individuals had $3 \%$ of the number of hypocretin cells found in control brains. By contrast, the other individual, who had received long-term opiate treatment for pain, showed $16 \%$ of the control number of these cells, and indeed had been re-diagnosed with idiopathic hypersomnia without cataplexy.

Together, these results imply that long-term use of opiate drugs may increase the number of hypothalamic hypocretin cells and might ameliorate certain symptoms of narcolepsy, such as cataplexy. These findings may also explain previous reports that people with narcolepsy are resistant to drug addiction, and could have implications for treating narcolepsy and opiate addiction.

Natasha Bray

ORIGINAL ARTICLE Thannickal, T. C. et al. Opiates increase the number of hypocretinproducing cells in human and mouse brain and reverse cataplexy in a mouse model of narcolepsy. Sci. Transl Med. 10, eaao4953 (2018) 